\title{
Die Arbeit des IAASB - Perspektiven und Probleme international einheitlicher Abschlussprüfungsstandards
}

\author{
Prof. Dr. Annette G. Köhler, Duisburg*
}

Inhaltsübersicht

ZGR 2015, 204-214

I. Einleitung und Auftrag des IAASB $\ldots \ldots \ldots \ldots \ldots \ldots \ldots \ldots$

II. Perspektive eins: Abschlussprüfungsstandards als Teilbereich des Normenkontextes für Abschlussprüfungen . . . . . . . . . . . . . . . . . . . . . . . 206

1. Problem .............................. 207

2. Lösungsansatz . . . . . . . . . . . . . . . . . . . . . 208

III. Perspektive zwei: Das IAASB als privater Standardsetter . . . . . . . . . . . . 209

1. Problem ............................. 210

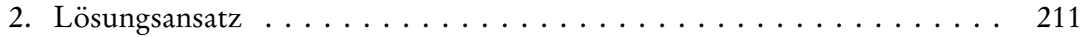

IV. Perspektive drei: Das IAASB als Förderer weltweit einheitlicher Abschluss-

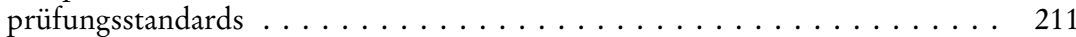

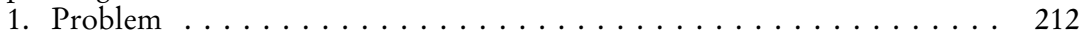

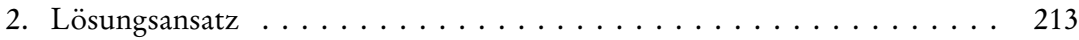

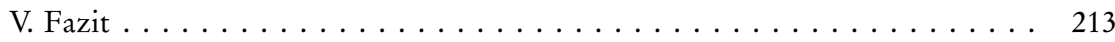

Im Mittelpunkt des vorliegenden Beitrags stehen drei Probleme, die aus Sicht der Verfasserin mit der Arbeit des IAASB als Herausgeber international einheitlicher Prüfungsstandards einhergehen: (1) die sog. Erwartungslücke in Bezug auf die Verantwortlichkeit des Abschlussprüfers und die damit verbundenen Implikationen für das IAASB, (2) die generell unterschiedlichen Anreize der von den herausgegebenen Standards betroffenen Stakeholder und daraus resultierende Unterschiede bei der Beteiligung der jeweiligen Stakeholder am Standardsetzungsprozess sowie (3) spezielle Unterschiede in den sprachlich und rechtsraumbedingten Anreizen der Stakeholder zur Mitwirkung bei der Entwicklung der Standards. Die Verfasserin zeigt jeweils problemspezifisch auf, inwiefern die Zusammensetzung des Boards, aber auch insbesondere die Arbeitsweise des Boards im Rabmen des sog. Due Process und deren Transparenz geeignet sind, diesen Problemen zu entgegnen und die Tätigkeit des IAASB im Hinblick auf eine Vertretung des öffentlichen Interesses zu fördern.

This paper focuses on three problems that, from the author's point of view, are inherent to the work of the IAASB as a body that promulgates international auditing standards: (1) the "expectations gap" related to the responsibilities of the auditor and the related implications for the IAASB; (2) the different incentives of stakeholders affected by the standards and resulting different participation by individual stakeholders in the standard setting process; and (3) specific language and jurisdictional differences in the incentives of individual stake-

* Die Verf. ist Inhaberin des Lehrstuhls für Rechnungswesen, Wirtschaftsprüfung und Controlling an der Universität Duisburg-Essen. 
bolders to participate in the standard setting process. The author shows how the IAASB's composition, and in particular, the IAASB's mode of operation as part of the IAASB's due process and the transparency thereof, appropriately address these problems and how these measures contribute to the IAASB's acting in the public interest.

\section{Einleitung und Auftrag des IAASB}

Im Mittelpunkt der folgenden Ausführungen stehen meine persönliche Wahrnehmung der Tätigkeit des International Audit and Assurance Standards Board (IAASB) und damit zumindest auch teilweise die Schilderung meiner eigenen Tätigkeit im Board und deren institutionellen Rahmenbedingungen. Ich werde auf die Tätigkeit des IAASB aus drei Perspektiven blicken - Perspektiven, die nach meiner Meinung aus Sicht der Öffentlichkeit, aber auch der Wissenschaft von besonderem Interesse sein dürften. Dabei werde ich jeweils im ersten Schritt das aus der Perspektive erkennbare zentrale, mit der Herausgabe international einheitlicher Abschlussprüfungsstandards durch das IAASB verbundene Problem herausarbeiten. In einem zweiten Schritt werde ich die korrespondierenden Lösungsansätze skizzieren, die die Tätigkeit des IAASB zu einem erheblichen Teil motivieren. Den Ausführungen vorangestellt seien Informationen zum Auftrag des IAASB; schließen werde ich mit einem Fazit. Der Beitrag ist nicht mit dem IAASB abgestimmt und gibt meine persönliche Meinung wieder.

Das IAASB ist ein unabhängiges Standardsetzungsgremium unter dem Schirm der International Federation of Accountants (IFAC). ${ }^{1}$ Zentrale Aufgabe des IAASB ist die Herausgabe von Standards für Prüfungs-, Bestätigungs- und verwandte Leistungen. Internationale Abschlussprüfungsstandards, die International Standards on Auditing (ISAs), stellen demnach lediglich einen Teilbereich der Verlautbarungen des IAASB dar. Im Mittelpunkt stehen dabei die Sicherstellung der Qualität der in Rede stehenden Leistungen sowie die Förderung deren internationaler Vergleichbarkeit vor dem Hintergrund des öffentlichen Interesses. Die Vertretung des öffentlichen Interesses manifestiert sich wie folgt:

- Die Zusammensetzung des IAASB ist divers: Es setzt sich aus neun praktizierenden Berufsträgern, sechs nicht praktizierenden Berufsträgern oder anderen Personen sowie drei sog. öffentlichen Mitgliedern zusammen.

- Die Tätigkeit des IAASB ist bestmöglich transparent: Alle Board-Sitzungen sind öffentlich; die Sitzungsunterlagen sind auf der Website des IAASB frei verfügbar.

1 Siehe für die folgenden Ausführungen http://www.ifac.org/anditing-assurance sowie KöHLER/Geirhofer, Zur Berichterstattung und Kommunikation des Abschlussprüfers nach den Vorschlägen des IAASB, in: iwp Wirtschaftsprüferjahrbuch 2014, 2014, S. 3 ff. 
- Die Tätigkeit des IAASB unterliegt der öffentlichen Aufsicht: Alle BoardSitzungen werden durch das Public Interest Oversight Board (PIOB) überwacht. $^{2}$

- Die Tätigkeit des IAASB wird durch eine sog. Consultative Advisory Group (CAG) begleitet. ${ }^{3}$

- Die Herausgabe der Standards folgt einem sog. Due Process. ${ }^{4}$

- Es erfolgt ein regelmäßiger Meinungs- und Erfahrungsaustausch mit den Stakeholdern des IAASB im Rahmen von Tagungen, Präsentationen, Diskussionen und Konsultationen.

\section{Perspektive eins: Abschlussprüfungsstandards als Teilbereich des Normenkontextes für Abschlussprüfungen}

In Bezug auf den Normenkontext der Abschlussprüfung kann vereinfachend zwischen den institutionellen Rahmenbedingungen der Abschlussprüfung einerseits und den fachtechnischen Normen, die das Vorgehen des Prüfers bei der Abschlussprüfung festlegen, andererseits differenziert werden. Erstere umfassen z. B. die Festlegung der Prüfungspflicht, des Prüfungsgegenstands und der Verantwortlichkeit für den Prüfungsgegenstand, Regelungen bezüglich des Zugangs zum Berufsstand der Wirtschaftsprüfer und Unabhängigkeitsanforderungen an den Abschlussprüfer, das relevante Haftungsregime und die Verantwortlichkeiten der Berufsaufsicht sowie anzuwendende Rechnungslegungsnormen. Letztere beziehen sich z. B. auf die Auftragsannahme, die Prüfungsplanung und -durchführung sowie die Dokumentation und Berichterstattung des Abschlussprüfers. Die Verpflichtung zur Anwendung der ISAs kann durch den Gesetzgeber oder im Rahmen einer freiwilligen Selbstverpflichtung erfolgen, nicht jedoch auf „Anweisung“ des IAASB.

Auf den ersten Blick erscheint die Differenzierung trivial, bei genauerer Betrachtung wird allerdings deutlich, wie eng die beiden Bereiche miteinander

2 Das PIOB wurde im Februar 2005 eingerichtet, um in erster Linie die Standardsetzungstätigkeiten unter dem Schirm der IFAC zu überwachen. Die acht Mitglieder werden von der internationalen Organisation der Börsenaufsichten, dem Baseler Ausschuss für Bankenaufsicht, der internationalen Vereinigung der Versicherungsaufsichten sowie der Weltbank nominiert. Zudem benennt die Europäische Kommission zwei Beobachter. Siehe auch http://www.ipiob.org/.

3 Die Vertreter der Mitgliedsorganisationen der CAG beraten das IAASB insbesondere bei der Festlegung des Arbeitsprogramms einschließlich der darin formulierten Prioritäten, der Initiierung und Durchführung der Projekte zur Verabschiedung von Standards sowie anderen relevanten Tätigkeiten. Die Arbeit der CAG wird durch das PIOB überwacht. Siehe auch http://www.ifac.org/auditing-assurance/cag.

4 Dieser umfasst: Research and consultation, transparent debate, exposure for public comment, consideration of comments received on exposure, affirmative approval. Siehe auch http://www.ifac.org/sites/default/files/downloads/IAASB_Fact_Sheet.pdf. 
verknüpft sind. So bezieht sich z. B. das Testat im Rahmen einer ISA-konformen Abschlussprüfung lediglich auf den geprüften Abschluss bzw. auf die diesen Abschluss konstituierenden Bestandteile wie Bilanz, Gewinn- und Verlustrechnung und Anhang. Das Interne Kontrollsystem oder das ComplianceSystem eines Unternehmens sind davon nur mittelbar und teilweise erfasst und stellen keine darüber hinausgehenden eigenständigen Prüfungsgegenstände dar. Im Bereich der Prüfung der sog. Going Concern-Prämisse des Unternehmens obliegt es nach ISA 700 dem Abschlussprüfer festzustellen, ob die Annahme der gesetzlichen Vertreter über die Fortführung der Unternehmenstätigkeit angemessen (d.h., die Voraussetzungen für den allgemeinen Bewertungsgrundsatz nach $\$ 252$ Abs. 1 Nr. 2 HGB gegeben) ist. Er hat ferner auf Basis der Prüfungsnachweise aus der Abschlussprüfung festzustellen, ob wesentliche Unsicherheiten hinsichtlich der Unternehmensfortführung (d.h., sog. bestandsgefährdende Risiken) vorliegen und diese ggf. im Abschluss zutreffend dargestellt sind. Da z. B. nach Einleitung eines Schutzschirmverfahrens gerade nicht (!) von der Liquidierung des Unternehmens ausgegangen werden kann, ist bei angemessener Darstellung der wesentlichen Unsicherheiten lediglich ein Zusatz in den Bestätigungsvermerk aufzunehmen - die Anwendung des allgemeinen Bewertungsgrundsatzes bleibt davon unberührt. Gegenstand der Abschlussprüfung ist es z. B. nicht, darüber hinausgehende Prüfungen der wirtschaftlichen Solidität des Unternehmens durchzuführen.

Im Gegensatz zu den beispielhaft dargestellten - vom Gesetzgeber festgelegten - Grenzen des Prüfungsgegenstands herrscht in der Öffentlichkeit immer wieder und insbesondere nach aufgedeckten Fraud-Fällen die Erwartung vor, dass im Rahmen der Abschlussprüfung auch ein Urteil über die Angemessenheit und Funktionsfähigkeit des Internen Kontrollsystems und des Compliance-Systems mit hinreichender Sicherheit abgegeben werde. Auch in Bezug auf die Going Concern-Prämisse kommt es in der Öffentlichkeit vornehmlich nach Unternehmenszusammenbrüchen zu Unverständnis, falls im Vorjahr ein uneingeschränkter Bestätigungsvermerk (mit oder ohne Zusatz) erteilt worden ist.

\section{Problem}

Die dargestellte Diskrepanz zwischen öffentlicher Erwartung an die Tätigkeit des Abschlussprüfers und der Wahrnehmung der Tätigkeit des Abschlussprüfers wird allgemein unter dem Begriff der „Erwartungslücke“ ${ }^{5}$ diskutiert. Diese kann auf ein unzulängliches Verständnis der Öffentlichkeit über die Tätigkeit des Abschlussprüfers (sog. Öffentlichkeitsversagen), die Nicht-Erfüllung der relevanten Normen durch den Abschlussprüfer (sog. Prüferver- 
sagen) oder die Unzulänglichkeit der relevanten Normen vor dem Hintergrund der durch die Öffentlichkeit motivierten Zielsetzung des Normensetzers (sog. Normenversagen) zurückgeführt werden.

In jedem Fall führt eine bestehende Erwartungslücke jedoch zu einer Verzerrung der Nutzenwahrnehmung der Abschlussprüfung und damit zur Gefahr einer ineffizienten Ressourcenallokation aufgrund von geprüften Abschlüssen. Dies kann zu einem Reputationsrisiko für den Setzer von Abschlussprüfungsstandards und damit des IAASB führen, was wiederum die Akzeptanz der vom IAASB verabschiedeten Normen und damit letztlich die Zielerreichung des IAASB beeinträchtigen kann. Die Sicherstellung der Qualität der Abschlussprüfung sowie die Förderung deren internationaler Vergleichbarkeit vor dem Hintergrund des öffentlichen Interesses sind in diesen Fällen gefährdet.

\section{Lösungsansatz}

Vor dem Hintergrund der dargestellten möglichen Folgen der Erwartungslücke nimmt das IAASB das gegenwärtige Projekt „Auditor Reporting“6 zum Anlass, zusätzliche Klarstellungen und Ergänzungen im Bestätigungsvermerk vorzusehen. So finden z.B. Erläuterungen der Verantwortlichkeiten von Management, Überwachungsorgan und Abschlussprüfer in den Bestätigungsvermerk Eingang. Diese umfasst auch eine Beschreibung der Verantwortlichkeiten des Managements in Bezug auf die oben erwähnte Going Concern-Prämisse sowie der damit verbundenen inhärenten Limitationen. Des Weiteren sucht das IAASB aktiv den Dialog zum IASB, um die „Prüfbarkeit“ von Rechnungslegungsinformationen zu fördern und damit einem Normenversagen vorzubeugen. So werden z. B. derzeit die auf die Going Concern-Prämisse bezogenen Ausführungen in IAS 1.25 mit dem IASB erläutert und Klarstellungen angeregt. Ebenso hat der Gesetzgeber auf europäischer Ebene im April 2014 neue Vorschriften zur Ausweitung der Berichterstattung des Abschlussprüfers im Bestätigungsvermerk verabschiedet.7 Folglich tritt das IAASB der Tatsache, dass ISAs lediglich einen Teilbereich des Normenkontextes darstellen und dies in der Öffentlichkeit durchaus zu einer Erwartungslücke führen kann, mit dem Bestreben möglichst umfassender Klarstellungen und zusätzlicher Transparenz im Bestätigungsvermerk - dem einzigen für die Öffentlichkeit sichtbaren direkten „Output“ der Abschlussprüfung - entgegen.

$6 \mathrm{Vgl}$. https://www.ifac.org/publications-resources/reporting-audited-financial-statements -proposed-new-and-revised-international.

7 Vgl. EU-Verordnung, Nr. 537/2014, Art. 10. 


\section{Perspektive zwei: Das IAASB als privater Standardsetter}

Zur Analyse möglicher mit der Entwicklung und Verabschiedung internationaler Abschlussprüfungsstandards durch das IAASB verbundener Probleme ist eine Differenzierung zwischen privater und staatlicher Normensetzung hilfreich. Hierzu soll in einem ersten Schritt auf eine - auch sprachlich präzisierende - Unterscheidung zwischen privater Regelsetzung einerseits und staatlicher Rechtsetzung andererseits in Anlehnung an Kirchner (2011) rekurriert werden. ${ }^{8}$ Demnach erfolgt aus prozessualer Sicht die staatliche Rechtsetzung primär durch Vorbereitung durch die Ministerialbürokratie und Modifikation durch das Parlament; der Einfluss von Interessengruppen ist lediglich indirekt möglich. Die Legitimierung der verabschiedeten Gesetze fußt auf der Akzeptanz der von dem Gesetz direkt und indirekt Betroffenen. Im Falle privater Regelsetzung steht die Modifikation von Entwürfen des Regelsetzers auf Basis der Stellungnahmen von Interessengruppen im Mittelpunkt - die Einflussnahme durch Interessengruppen ist explizit Bestandteil des Prozesses. Eine demokratische Legitimierung ist letztlich nicht möglich, die Legitimierung ergibt sich aus dem Prozess selbst, d. h. aus der Verabschiedung der Regeln durch das Gremium.

Nach Ansicht von Kirchner kommt es bei der privaten Regelsetzung im Gegensatz zur staatlichen Rechtsetzung zu negativen externen Effekten der Regeln, da Insider als Mitglied des privaten Regelsetzers ihren Einfluss zulasten Dritter geltend machen können. ${ }^{9}$ Ferner ist jedoch auch die Übernahme privater Regeln in Recht durch den staatlichen Regelsetzer möglich. In diesem Fall lassen sich durch Modifikationen der Regeln negative Externalitäten vermeiden. Aus einer praktischen Perspektive können die von Kirchner den beiden alternativen Prozessen zugeschriebenen Eigenschaften allerdings auch anders wahrgenommen werden. So stellt sich die Frage, ob sich die Legitimierung des staatlichen Rechtsetzungsprozesses nicht eher aus der Legitimität des Rechtsetzers ergibt - unabhängig von der Akzeptanz des durch den Rechtsetzer verabschiedeten Rechts - und ob Akzeptanz nicht vielmehr die „Quasi“-Legitimierung privater Regelsetzung ermöglicht. Eine demokratische Legitimierung privater Regeln dürfte lediglich durch die Übernahme der Regeln in Recht durch den staatlichen Rechtsetzer gelingen. ${ }^{10}$ Beispiel hierfür wäre die Übernahme der ISAs durch den europäischen Gesetzgeber. Außerdem lässt sich am Beispiel der Verabschiedung des Bilanzrechtsmodernisierungsgesetzes demonstrieren, dass auch der staatliche Rechtsetzer seinem Auftrag durch die Veröffentlichung von Entwurfsfassungen und deren

8 Vgl. Kirchner, Erasmus Law Review 2011, 163.

9 Vgl. Kirchner, Erasmus Law Review 2011, 169.

10 Diese Vorgehensweise wird auch als „hybride Regelsetzung“ bezeichnet. Vgl. KIRCHNER/SCHMIDT, BFuP 2006, 388. 
Modifikation in Reaktion auf die Stellungnahmen von Interessengruppen nachkommt. ${ }^{11}$

\section{Problem}

Auch wenn bei staatlicher Rechtsetzung vom Einfluss betroffener Interessengruppen ausgegangen werden kann, so ist dieser zumindest aus legalistischer Sicht vermeidbar. Für den privaten Regelsetzungsprozess ist er hingegen konstituierend. In Bezug auf die Verlautbarung der ISAs durch das IAASB kommt dies in der Ausgestaltung des sog. „Due Process“ zum Ausdruck. Demnach ist jeder Standard-Entwurf auf der Website des IAASB zur Einholung von Stellungnahmen zu veröffentlichen (sog. „exposure for public comment“). Die Kommentierungsfrist beträgt mindestens 120 Tage. Die Stellungnahmen und Vorschläge zum Entwurf sind im Rahmen mindestens einer öffentlichen Sitzung des IAASB zu diskutieren und der Entwurf gegebenenfalls anzupassen (sog. „consideration of comments received on exposure"). Falls die Änderungen vom IAASB als so wesentlich angesehen werden, dass ein weiterer Entwurf erstellt werden muss (sog. re-exposure draft), ist dieser ebenfalls für eine Kommentierung vorzusehen. Auch wenn das IAASB in erster Linie dem öffentlichen Interesse, d. h. einem Interessenausgleich verpflichtet ist, so ist gleichwohl den auf einen Standard-Entwurf Antwortenden explizit Rechnung zu tragen.

Würden alle sowohl positiv als auch negativ durch einen Standard Betroffene gleichermaßen ihre Positionen in Stellungnahmen vertreten, wäre eine auf einen Interessenausgleich abzielende Ausgestaltung der Standards zwar immer noch nicht trivial, aber zumindest für Dritte relativ einfach nachvollziehbar. Tatsächlich ist das Feedback auf Standard-Entwürfe jedoch systematisch verzerrt - und genau hierin liegt das Problem: Üblicherweise fallen die mit Abschlussprüfungsstandards verbundenen Nutzeneffekte indirekt und zeitverzögert an, da sich die Glaubwürdigkeit von Abschlussinformationen erst im Laufe der Zeit manifestiert und - infolge des Mangels an einer nicht-glaubwürdigen Referenzinformation - in ihrem Nutzen unterschätzt wird. Die mit Abschlussprüfungsstandards verbundenen Kosten fallen üblicherweise jedoch zeitnah und direkt messbar ins Gewicht - nämlich auf Seiten der Abschlussprüfer und - sofern die Kostenerhöhungen weitergegeben werden können auf Seiten der Abschlussersteller bzw. der Eigentümer der geprüften Unternehmen. Folglich ist der Anreiz zur Vertretung des eigenen Interesses - vereinfachend ausgedrückt - auf der Seite der Abschlussadressaten niedriger als auf der Seite der Abschlussprüfer, sodass es nicht verwundern kann, wenn die Anzahl der Stellungnahmen aus dem Kreis der Abschlussprüfer und deren

11 Siehe auch Richardson, Accounting, Organizations and Society 2009, $571 \mathrm{ff} ; \mathrm{ZüLCH} /$ Gebhardt/Hoffmann, J Betriebswirtsch 2009, 4 ff. 
Berufsstandorganisationen die Anzahl der Stellungnahmen von Abschlussadressaten um ein Vielfaches übersteigt. Allein die zu erwartenden Verfügbarkeitsheuristiken machen die potentiellen Schwierigkeiten bei dem auf das öffentliche Interesse ausgerichteten Vorgehen des IAASB deutlich.

\section{Lösungsansatz}

Das IAASB setzt an der Vermeidung von Verfügbarkeitsheuristiken ${ }^{12}$ an. Dies lässt sich anhand des Vorgehens beim bereits o.g. Auditor Reporting-Projekt aufzeigen: Über die im Due Process vorgesehenen Vorstufen zum eigentlichen Standardsetting hinaus hat das IAASB eine außerordentlich breit angelegte, mehrere Stufen umfassende öffentliche Konsultation ${ }^{13}$ sowie zahlreiche Fachtagungen und Diskussionsrunden in Europa, den USA, aber auch im süd-ostasiatischen Raum zur möglichen Weiterentwicklung der Berichterstattung des Abschlussprüfers durchgeführt. Hierzu wurden explizit diverse Vertreter von Abschlussadressaten, z. B. Investoren und Finanzanalysten, aber auch von Berufsaufsichten eingeladen. Ergebnisprotokolle und Berichte der Veranstaltungen flossen explizit in die Sitzungsunterlagen ein und wurden auch auf den IAASB-Sitzungen vorgetragen und erörtert. Auf diese Weise wurde Informationsbedürfnissen und potentiellen Nutzeneffekten möglicher Erweiterungen der Berichterstattung explizit Raum gegeben. Inhalt und Ausgewogenheit der Erörterungen sind durch Sicherstellung der Öffentlichkeit der Sitzungen auch für interessierte Dritte stets nachprüfbar.

\section{Perspektive drei: Das IAASB als Förderer weltweit einheitlicher Abschlussprüfungsstandards}

Das IAASB verfolgt u. a. die Zielsetzung, die Leistungserstellung und Berichterstattung im Rahmen der Abschlussprüfung international vergleichbar zu machen. Das Mittel der Wahl ist die Verlautbarung von Standards, die von möglichst vielen Mitgliedsorganisationen der IFAC oder Gesetzgebern in nationale (oder möglicherweise im Fall Europas: supranationale) Prüfungsstandards übernommen werden - also die Ablösung verschiedener nationaler Prüfungsstandards durch die ISAs. Folgt man Kirchner (2011), so bedeutet die damit verbundene und vor allem bei privaten Regelsetzern verbreitete Harmonisierung nichts anderes als eine Kartellbildung nationaler Regelsetzer, d. h. die Ausschaltung regulatorischen Wettbewerbs und somit schließlich die Ent-

12 Siehe auch Tversky/Kahneman, Cognitive Psychology 1973, $207 \mathrm{ff}$.

13 Im Mai 2011 wurde ein erstes Konsultationspapier „Enhancing the Value of Auditor Reporting: Exploring Options for Change“ vorgelegt; dem folgte im Juni 2012 die „Invitation to Comment: Improving the Auditor’s Report“. 
wicklung suboptimaler Regeln. ${ }^{14}$ Demgegenüber - so Kirchner - befinden sich nationale staatliche Rechtsetzer im internationalen regulatorischen Wettbewerb, der letztlich auch den (eigenmotivierten) diskretionären Handlungsspielraum des Rechtsetzers eindämmt. ${ }^{15}$ Diese Auffassung ist aus legalistischer Sicht durchaus nachvollziehbar. Unter ökonomischem Blickwinkel stellt sich hierbei allerdings die Frage, inwieweit die Bedingungen für einen regulatorischen Wettbewerb überhaupt erfüllt sind.

Voraussetzung für internationalen regulatorischen Wettbewerb ist die freie, d. h. kostenlose, Auswahl der Nachfrager, d.h. die durch ein Gesetz Betroffenen, zwischen regulatorischen Regimes oder die kostenlose Änderung regulatorischer Regimes im Zeitablauf. Im ersten Fall können sich die Betroffenen frei zwischen alternativen Gesetzen entscheiden und so die diversen Rechtsetzer belohnen oder bestrafen. Im zweiten Fall wählen die Betroffenen den Rechtsetzer bei nächster Gelegenheit ab und wählen stattdessen einen anderen Rechtsetzer, der unverzüglich andere Gesetze erlässt. Nur so setzen sich langfristig die „besten“ Gesetze durch. Allein die Schilderungen dürften deutlich machen, dass die Transaktionskosten im ersten Fall i. d. R. prohibitiv hoch sind, da mit dieser Wahl z. B. ein grenzüberschreitender Standortwechsel einherginge. Auch der zweite Fall ist z.B. aufgrund von Pfadabhängigkeiten nicht realistisch. Im Gegenteil: Harmonisierung von Regel- oder Rechtssystemen ist gerade die $R e-$ aktion auf Transaktionskosten, die mit dem Wechsel zwischen Regel- und Rechtssystemen oder mit gleichzeitiger Anwendung mehrerer Regel- und Rechtssysteme einhergehen. ${ }^{16}$ Findet diese Harmonisierung nicht durch Kooperation und Konvergenz aufSeiten der Regel-oder Rechtsetzer statt, schaffen die durch Regeln oder Gesetze Betroffenen Fakten:So hat sich z. B. eine Gruppe von Wirtschaftsprüferpraxen, die in internationale Netzwerke eingebunden sind und grenzüberschreitende Abschlussprüfungen anbieten, mittlerweile freiwillig dazu verpflichtet, die ISAs anzuwenden (sog. „Forum of Firms“) ${ }^{17}$.

\section{Problem}

Die mit der Entwicklung weltweit einheitlicher und (faktisch zwingend) in der Originalfassung in englischer Sprache verfasster Abschlussprüfungsstandards einhergehenden Probleme liegen vielmehr darin, dass die Interessenlagen der durch die Standards Betroffenen nicht nur funktional, sondern auch national

14 Vgl. Kirchner, Erasmus Law Review 2011, 167.

15 Vgl. Kirchner, Erasmus Law Review 2011, 166.

16 Zur Einordnung und Analyse dieses Harmonisierungseffektes sowie der damit verbundenen Nutzen- und Kosteneffekte vgl. KöHLER/Merкт/BöHm, Evaluation of the Possible Adoption of International Standards on Auditing (ISAs) in the EU, 2009, http://duepublico.uni-duisburg-essen.de/servlets/DocumentServlet?id=21502.

$17 \mathrm{Vgl}$. http://www.ifac.org/about-ifac/forum-firms. 
geprägt sein können, und somit ein vor dem Hintergrund der Vertretung des öffentlichen Interesses angemessener Interessenausgleich komplexer und anspruchsvoller ist als ein Interessenausgleich auf nationaler Ebene. Hinzu kommt, dass die ISAs auf einer Kunstsprache basieren, die bei einer Übersetzung die Interpretation der Begriffe besonders notwendig macht. Angesichts der Interdependenzen zwischen kulturellem und institutionellem Umfeld einerseits und Sprachgebrauch andererseits können national geprägte Präferenzen für bestimmte Formulierungen in den ISAs nicht überraschen. Umgekehrt können Übersetzungen Freiheiten eröffnen, die in englischsprachigen Ländern nicht gegeben sind.

Damit kommen die bereits in Abschnitt III. 1. beschriebenen Selektionseffekte bei der Formulierung der Interessen ins Spiel: Stakeholder aus Ländern, in denen die ISAs unmittelbare Anwendungsverpflichtung besitzen und deren Amtssprache Englisch ist, haben einen stärkeren Anreiz, ihr Interesse geltend zu machen, als Stakeholder aus Ländern, in denen die ISAs allenfalls für die Prüfung ausgewählter Unternehmen anzuwenden sind oder lediglich als Leitlinie für die Entwicklung eigener nationaler Prüfungsstandards dienen und deren Amtssprache nicht Englisch ist. Bezeichnenderweise sind beide Effekte interagierend. So ist z. B. der Verpflichtungsgrad der ISAs in Australien, Canada und UK im Vergleich zum Verpflichtungsgrad der ISAs in Japan und China außerordentlich hoch.

\section{Lösungsansatz}

Wie bereits unter Abschnitt III. 2. aufgezeigt, tritt das IAASB den inhärent asymmetrischen Anreizen der Interessenvertretung und damit potentiell verbundenen Verfügbarkeitsheuristiken durch eine proaktive Einbindung diverser regionaler oder nationaler Vertreter entgegen. Neben der auch unter regionalen Aspekten diversen Zusammensetzung des Boards sind z. B. derzeit die drei ständigen Beobachter (sog. Observer) mit Rederecht die Vorsitzende der CAG, ein Vertreter der Europäischen Kommission und ein Vertreter der japanischen Aufsichtsbehörde für Finanzdienstleistungen. Auch der Vertreter des PIOB hat eine explizit supranationale Perspektive einzunehmen. Zudem werden bereits im Standardsetzungsprozess nationale institutionelle Besonderheiten und potentielle Übersetzungsprobleme thematisiert. Auf diese Weise werden die Voraussetzungen für eine auch aus internationaler Sicht ausgewogene Diskussion und Meinungsbildung geschaffen.

\section{Fazit}

Mit dem IAASB wurde ein privater Standardsetzer geschaffen, der u. a. international einheitliche Abschlussprüfungsstandards herausgibt und auf diese 
Weise einen wesentlichen Beitrag zu einer einheitlich hohen Prüfungsqualität und einer internationalen Vergleichbarkeit der Leistungserstellung und Berichterstattung des Abschlussprüfers leistet. Die in Abhängigkeit von der gewählten Perspektive mit dem Auftrag und der Tätigkeit des IAASB einhergehenden Probleme sowie die vom IAASB aktiv verfolgten korrespondierenden Lösungsansätze waren Gegenstand der obigen Ausführungen. Abschließend lässt sich Folgendes feststellen:

- Transparenz und Kommunikation zur Klarstellung der Verantwortlichkeiten des IAASB sind v. a. angesichts der Diskussion über die Ausdehnung des Prüfungsgegenstands wichtiger denn je.

- Für die Legitimierung der ISAs ist letztlich deren Übernahme in nationales oder supranationales Recht im Rahmen eines legitimierten Prozesses erforderlich.

- Die Negierung des Einflusses von Interessengruppen kann weder in Bezug auf staatliche Rechtsetzung noch private Regelsetzung überzeugen. Entscheidend für die Glaubwürdigkeit und damit Akzeptanz von Gesetzen und Regeln sind Transparenz und Nachvollziehbarkeit der Entscheidungen innerhalb des Recht- bzw. Regelsetzungsprozesses.

- International verschiedene Abschlussprüfungsstandards sind in Anbetracht der Transaktionskosten von Abschlussadressaten, -prüfern und -erstellern keine Alternative zu international einheitlichen Abschlussprüfungsstandards. 


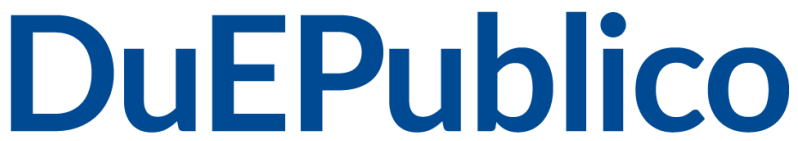

\section{Duisburg-Essen Publications online}

UNIVERSITÄT

DE USSEN R R G

offen im Denken

Ub $\mid \begin{gathered}\text { universitäts } \\ \text { bibliothek }\end{gathered}$

Dieser Text wird über DuEPublico, dem Dokumenten- und Publikationsserver der Universität Duisburg-Essen, zur Verfügung gestellt. Die hier veröffentlichte Version der EPublikation kann von einer eventuell ebenfalls veröffentlichten Verlagsversion abweichen.

DOI: $\quad 10.1515 / \mathrm{zgr}-2015-0204$

URN: urn:nbn:de:hbz:464-20200529-083423-2

Alle Rechte vorbehalten.

Dieser Beitrag ist mit Zustimmung des Rechteinhabers aufgrund einer (DFG-geförderten) Allianz- bzw. Nationallizenz frei zugänglich. 